\title{
An Algorithm for Target Identification of Fanconi Anemia in Drug Discovery
}

\author{
Md Jayedul Haque \\ Dept. of Computer Science \& \\ Engineering \\ United International University
}

\author{
Galib Muhammad Shahriar \\ Dept. of Computer Science \& \\ Engineering \\ United International University
}

\author{
Kh. Abdullah Al- Aff \\ Dept. Of Microbiology and \\ Immunology \\ Bangladesh University of Health \\ Sciences
}

\begin{abstract}
At the current age, Bioinformatics and Computational Biology which is the field of science, includes not only Molecular biology but also Computer science and Databases, Information technology or Information management. The objective of this field is to develop methods and software tools for analyzing and interpreting biological data. The study of Computational Biology and Bioinformatics not only developing the phase of science but also developing so many cure of malignant and remarkable diseases in human span. For example, DNA replication can be considered the primal one. DNA replication causes cell division but when this is thwarted the unusual malady takes place. Fanconi Anemia is one of the unusual disorders which arises by the means of blockage of DNA replication. The subsequent outcome of Fanconi Anemia is cancer which is sometimes exacerbated and no other remedy found even along with drug. So, it is becoming vital to identify and finding of drug discovery methodology of Fanconi Anemia. Enormous research has been introduced to Fanconi Anemia which implicates the reason and clinical research. In this study we are going to propose an algorithm which will be able to identify the presence of Fanconi Anemia and will make a space to identify the target of drug discovery.
\end{abstract}

\section{Keywords}

Algorithm, Fanconi Anemia, DNA, Drug Discovery, Target Identification, Mutation.

\section{INTRODUCTION}

In recent time, it has been shown in several researches on the human genome that although many frequently occurring diseases are normally caused by inheritance of mutations in multiple genes at once, these types of diseases can also be caused by rare hereditary mutations in a single gene. This problem is implicated as Genetic disorders. Genetic Disorder causes mutation, residual DNA replication and lack of DNA replication. It can be exemplified the lack of replication in host body which causes diseases like Ataxia telangiectasia (AT) and Fanconi anemia (FA). The effects of Fanconi anemia are: 1.the majority develop cancer, 2.most often acute myelogenous leukemia, 3. $90 \%$ develop bone marrow failure (the inability to produce blood cells) by age 40. Fanconi anemia is a reason of affecting several parts of the body. Those people who have this condition may have bone marrow failure, physical abnormalities, organ defects, and an increased risk of certain cancers.

The main functionality of bone marrow is to produce new blood cells. These include red blood cells, which carry oxygen to the body's tissues; white blood cells, which fight infections; and platelets, which are necessary for normal blood clotting. Around 90 percent of people who are affected with Fanconi anemia have impaired bone marrow condition that leads to a wan in the production of all blood cells (aplastic anemia). Affected people suffer from extreme tiredness (fatigue) because of low numbers of red blood cells (anemia), frequent infections because of low numbers of white blood cells (neutropenia), and clotting problems because of low numbers of platelets (thrombocytopenia). Fanconi anemia may also develop myelodysplastic syndrome, a condition in which immature blood cells fail to develop normally among affected people. More than half of the people have physical abnormalities with Fanconi Anemia. Irregular skin coloring such as unusually light-colored skin (hypopigmentation) or café-au-lait spots, which are flat patches on the skin that are darker than the surrounding area is involved by these abnormalities. According to the U.S. National Library of Medicine other possible symptoms of Fanconi anemia include malformed thumbs or forearms and other skeletal problems including short stature; malformed or absent kidneys and other defects of the urinary tract; gastrointestinal abnormalities; heart defects; eye abnormalities such as small or abnormally shaped eyes; and malformed ears and hearing loss. People with this condition may have abnormal genitalia or malformations of the reproductive system. As a result, most affected males and about half of affected females cannot have biological children (are infertile). Additional signs and symptoms can include abnormalities of the brain and spinal cord (central nervous system), including increased fluid in the center of the brain (hydrocephalus) or an unusually small head size (microcephaly).People with Fanconi anemia have a waxed hazard of provoking a cancer of blood-forming cells in the bone marrow called acute myeloid leukemia (AML) or tumors of the head, neck, skin, gastrointestinal system, or genital tract. The likelihood of developing one of these cancers in people with Fanconi anemia is between 10 and 30 percent.

\section{LITERATURE REVIEW}

Fanconi anemia (FA) is a part of a group of inherited bone marrow failure disorders that was initially described in 1927 by Guido Fanconi. FA is a heterogeneous group of diseases with variable genotypes and phenotypes with defective DNA repair mechanisms. Many years after Fanconi's report it became evident that defects in several genes can cause Fanconi anemia. Almost 40 years after Fanconi's report it was Traute Schroeder who noticed spontaneous chromosomal breakage during routine cytogenetic analysis of FA patients [1], which classified FA as a chromosomal instability syndrome. Ten years later, Masao Sasaki discovered that the chromosomal abnormalities in FA cells were drastically enhanced by the DNA cross-linking agent mitomycin C (MMC) [2]. This observation has provided the basis for the widely used diagnostic chromosomal breakage test, as first introduced by Arleen Auerbach [3]. The vast majority of FA patients present in childhood when hematopoietic disease, be 
it bone marrow failure or acute myeloid leukemia, predominates. The key treatment of such patients has been stem cell transplantation (SCT) [4]. Hematopoietic stem cell transplantation (HSCT) is the only treatment with a curative potential for the hematologic manifestations in this severe congenital disease [5-7]. Early experience with HSCT in the treatment of FA was unsuccessful because of the basic molecular defect in these patients: an excessive sensitivity to cytotoxic agents that led to severe radiation and chemotherapy-related toxicity, and consequently, a high rate of treatment-related mortality (TRM) as well as unusually severe graft-versus-host disease (GVHD) [8, 9]. In 2011 Forty-one patients with FA (16 males, 25 females) who underwent 46 HSCTs in the 3 participating medical centers from June 1993 until September 2007 were included in this retrospective study. They used some statistical method like the Kaplan-Meier method to plot the probabilities of engraftment and OS, the significance was estimated by logrank test and logistic regression analysis and Chi-square was used to analyze some survival predictors [10]. To elucidate the molecular defect in FA cells, several labs started to identify the gene defects in the various FA complementation groups. In 1992, Manuel Buchwald's lab identified the first FA gene, FANCC, using a home-made episomal cDNA expression library and the FA-C lymphoblast line HSC536 [11]. This gene cloning method turned out to be quite powerful as it subsequently led to the discovery of FANCA, FANCE, FANCF, and FANCG in their lab [12-14]. The core complex, with FANCL as the catalytic subunit, acts as an E3ubiquitin ligase to monoubiquitinate FANCD2 and FANCI in conjunction with the E2-conjugating enzyme UBE2T [15]. Ubiquitin is removed from FANCD2 by the deubiquitinating enzyme USP1 [16]. An improved predictive machine learning approach using the feature selection technique and two class Linear Discriminant Technique (LDA) algorithm to accurately predict the active novel USP1/UAF1 inhibitor compounds [17]. XPF-ERCC1 Acts in Unhooking DNA Interstrand Crosslinks in Cooperation with FANCD2 and FANCP/SLX4 and XPF depletion, but not MUS81 or FAN1 depletion, abrogates ICL repair [18]. To identify the target and validation of drug discovery many machine learning tools are used in bioinformatics [19]. In this study, an algorithm to identify the Fanconi Anemia has been proposed.

\section{BACKGROUND}

This section provides the necessary background on the problem. The difficulties are briefly explained along with the proposal.

\subsection{The Problem}

Fanconi anemia is most often inherited in an autosomal recessive pattern, which means both copies of the gene in each cell have mutations According to the U.S. National Library of Medicine. Mutations in at least 15 genes can cause Fanconi anemia. These genes generate proteins which are engaged in a cell process known as the FA pathway. The FA pathway is activated when the process of making new copies of DNA which is called DNA replication and blocked due to DNA damage. The FA pathway emits certain proteins to the area of damage, which triggers DNA repair so that DNA replication can be sustained. The FA pathway is particularly responsive to a certain type of DNA damage acquainted as interstrand cross-links (ICLs). The time of occurring ICLs is when two DNA building blocks (nucleotides) on opposite strands of DNA are abnormally attached or linked together, which stops the process of DNA replication [20,21].The motive of ICLs is building up of toxic substances which are generated in the body or by treatment with certain cancer therapy drugs [22].

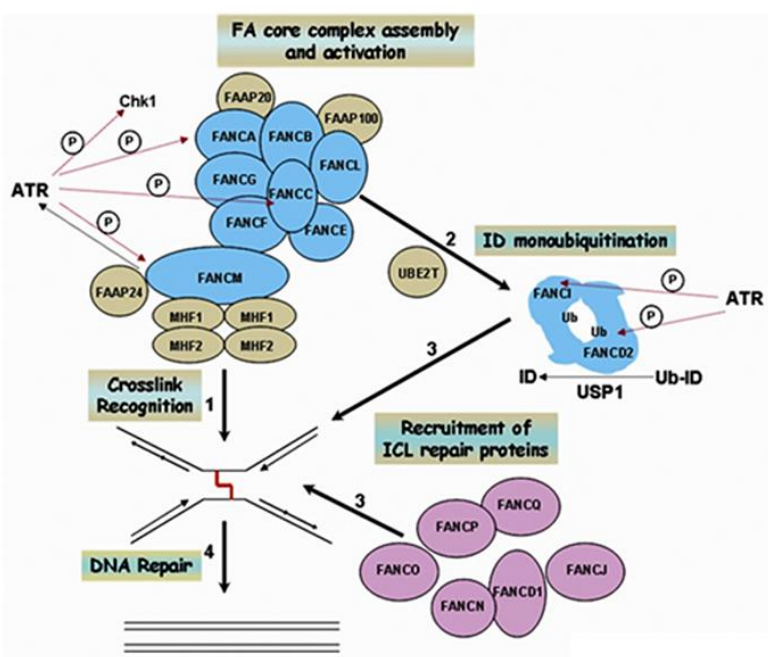

Fig 1: Structure of FA Pathway [23]

Eight proteins linked with Fanconi anemia group together to form a complex known as the FA core complex. Two proteins called FANCD2 and FANCI are activated by the FA core complex [25-28]. The activation of these two proteins invoke DNA repair proteins to the area of the ICLs so that the crosslink can be removed and DNA replication can sustain.

According to the U.S. National Library of Medicine $80 \%$ to 90\% of cases of Fanconi Anemia are due to mutations in one of three genes, FANCA, FANCC, and FANCG. These genes provide instructions for generating material of the FA core complex. Mutations in any of the many genes associated with the FA core complex will cause the complex to be nonfunctional and disrupt the entire FA pathway. As a result, DNA damage is not repaired efficiently and ICLs grow up over time. The ICLs stop DNA replication, ultimately resulting in either abnormal cell death due to an inability to make new DNA molecules or uncontrolled cell growth due to a lack of DNA repair processes. Cells that divide quickly, such as bone marrow cells and cells of the developing fetus, are individually affected. The death of these cells results in the decrease in blood cells and the physical abnormalities characteristic of Fanconi Anemia. When the building up of errors in DNA leads to uncontrolled cell growth, affected individuals can develop acute myeloid leukemia or other cancers. Eventually, FA is the result of a genetic defect in a cluster of proteins responsible for DNA repair [24].

\subsection{Proposed Work}

This study proposes a very tractable approach of identifying the target of Fanconi Anemia in Drug Discovery. So many research paper have been reviewed on Fanconi Anemia to find out the methodology and its validation. Fanconi Anemia occurs in 1 in 160,000 individuals ubiquitous. This condition is more common among people of Ashkenazi Jewish descent, the Roman population of Spain, and black South Africans. In this study an algorithm to identify the Fanconi Anemia has been proposed. After the proposal all the processes have been described briefly which implies the involvement of Computer Science in this premises. Not only proposal but also introduction to online tools has been occupied in this study. 


\section{METHODOLOGIES}

During DNA replication and blockade due to DNA damage, FA pathway is activated which sends protein to the damaged DNA to repair. Cells derived from Fanconi anemia (FA) patients are hypersensitive to agents that induce DNA interstrand cross-links (ICLs) and exhibit ICL-induced chromosomal instability. As a result DNA replication hampered. The encoded gene in FA pathway have been grouped into three categories: (1) the FA core complex, including the E3 ligase, FANCL; (2) the ID2 complex, the substrate for the E3 ubiquitin ligase activity of the core complex; and (3) downstream proteins that possess a DNA repair or damage tolerance function.
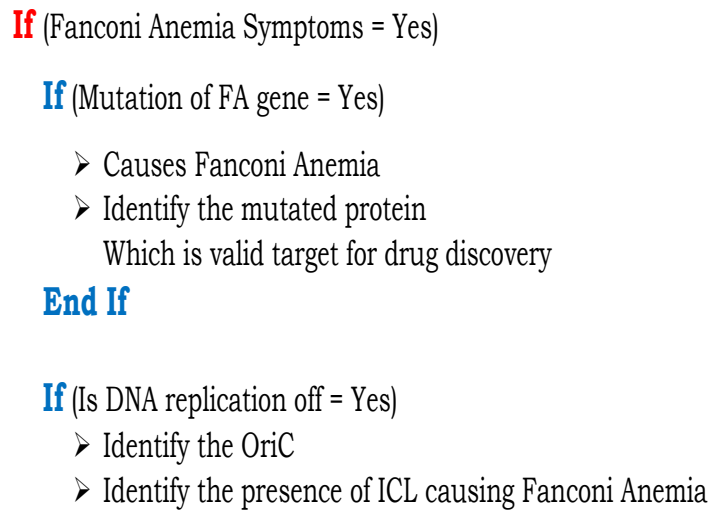

$$
\begin{aligned}
& \text { If (Is ID2 Complex activated = Yes) } \\
& \text { If (UBE2T Protein<regular amount) } \\
& \text { Target found for drug discovery } \\
& \text { End If }
\end{aligned}
$$

End If

> Check Depletion level of XPF with respect to MUS81 and FAN1

\section{If $($ Check XPF - ERCC1 defected $=$ Yes) \\ Causes Fanconi Anemia which is the target found for drug discovery}

\section{End If}

\section{End If}

\section{End If}

Fig 2: Algorithm of Target identification of Fanconi Anemia

Core complex is essential for the monoubiquitination of the FA proteins FANCD2 and FANCI, however, the procedure by which ubiquitylated FANCI-FANCD2 improves these incisions remains unknown. A number of nucleases have been proposed to contribute in ICL repair, including MUS81EME1, XPF-ERCC1, FAN1, SLX4- SLX1, SNM1A, XPG, and FEN1. Their accurate roles have not been identified, but their activity is likely considerable at various stages of ICL repair, including unhooking, $\mathrm{HR}$, and processing. XPF recruitment depends on SLX4 and recruitment is facilitated by FANCD2-ub (by Puck Knipsheer). Sometimes, XPF-ERCC1 defects causes Fanconi Anemia. Algorithm 1 proposes how Target identification of Fanconi Anemia should work.

\subsection{Mutations of FA Gene}

A gene mutation is an enduring alteration in the DNA sequence that makes up a gene, such that the sequence differs from what is found in most people. Mutations range in size; they can affect anywhere from a single DNA building block (base pair) to a large segment of a chromosome that includes multiple genes.

\subsubsection{Substitution}

A substitution is a mutation that exchanges one base for another (i.e., a change in a single "chemical letter" such as switching an A to a G).

\subsubsection{Insertion}

Insertions are mutations in which extra base pairs are inserted into a new place in the DNA.

\subsubsection{Deletion}

Deletions are mutations in which a section of DNA is lost, or deleted.

Jpred v.4 is the latest version of the popular JPred Protein Secondary Structure Prediction Server [41] which provides predictions by the JNet algorithm, one of the most accurate methods for secondary structure prediction [42], despite its apparent simplicity, the problem of quantifying the differences between two structures of the same protein or complex is non-trivial and continues evolving. In this section, several methods have been described which are routinely used to compare computational models to experimental answers in several modeling assessments [43].

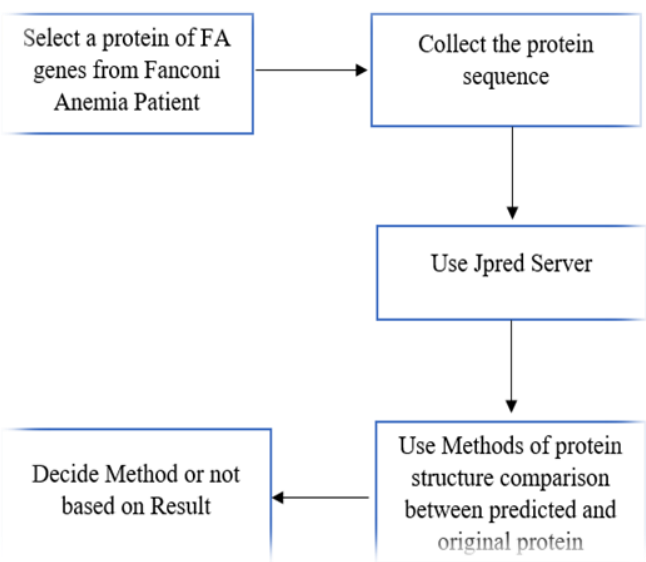

Fig 3: Process of Identify Mutation

\subsection{Identify the OriC}

In molecular biology, DNA replication is the biological process which reproduces two new replicas of DNA from one original DNA molecule. The replication begins in a region called the origin of replication (OriC). The OriC region is always located within the intergenic region. Three methods are used to identify the OriC DNA asymmetry, DNA box distribution, DNA gene location. The tool Ori Finder has been used to find out the OriC [44].

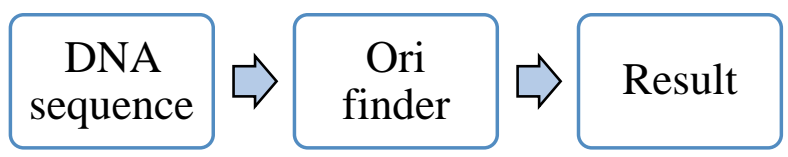

Fig 4: Process of OriC finding 


\subsection{Identify the Presence of ICL}

In genetics, crosslinking of DNA takes place when several exogenous or endogenous agents react with two different positions in the DNA. This can take place in either the identical strand (intrastrand crosslink) or in the opposite strands of the DNA (interstrand crosslink). Crosslinks also take place between DNA and protein. DNA replication is thwarted by crosslinks, which cause replication stall and cell death if the crosslink is not repaired. Although the FA pathway might play a minor role in ICL repair during G1 phase [30, 31], its primary function is exerted in S phase [3235]. These results suggest that ubiquitylated FANCI, FANCD2 controls ICL repair in S phase, but the underlying mechanism is unknown. The FANCM/FAAP24 sub-complex is considered to act as a "molecular sensor", recognizing a replication fork stalled at an ICL and invoking downstream material of the ICL repair machinery. FANCM and its adjusted proteins FAAP24, MHF1 and MHF2 (FANCMinteracting histone fold proteins 1 and 2) bind preferentially to branched DNA structures in vitro [36-38] and FANCMdepleted cells fail to invoke the Fanconi core complex or to mono-ubiquitylate the FANCD2-FANCI heterodimer, key events that occur during normal ICL repair [39].

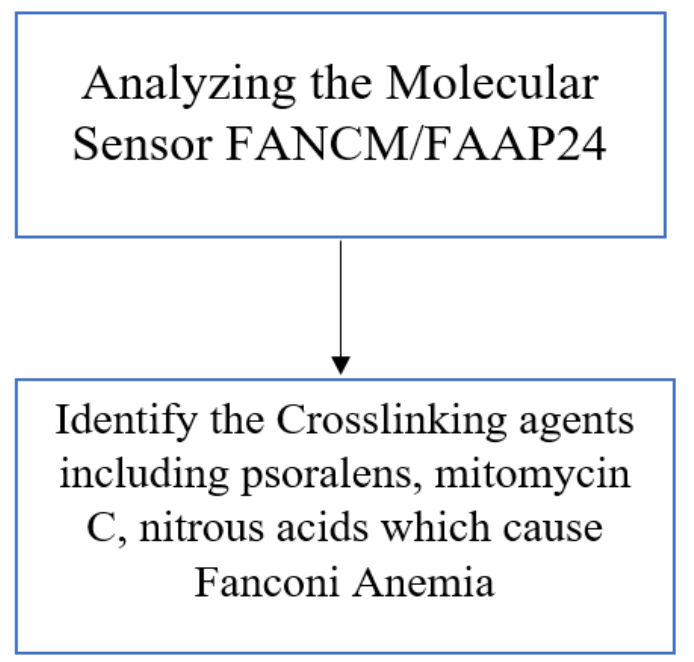

Fig 5: Methodologies to ICL Recognition

\subsection{ID2 Complex Activation}

FA/BRCA pathway is activated during the DNA replication period which invoke the FA core complex. The FA core complex which illustrate the upstream part of the pathway, form of FANCA, -B, -C, -E, -F, -G, -L, -M, and two FAassociated proteins FAAP24 and FAAP100. This complex is important for the mono-ubiquitination of the FA proteins FANCD2 and FANCI which is called the ID2 complex and responsible to invoke downstream proteins including SLX4 and XPF-ERCC1. In this reaction, FANCL is the E3 ubiquitin ligase and UBE2T is the E2-conjugating enzyme. Monoubiquitinated FANCD2 and FANCI co-localize in damageinduced nuclear foci with BRCA2, which, together with its binding partner PALB2 and BRIP1 belong to the downstream branch of the pathway. Ubiquitin is removed from FANCD2 and FANCI $b$ the deubiquitinating enzyme USP1 [22]. Sometimes, deficiency of UBE2T causes Fanconi anemia because UBE2T is responsible for ubiquitination of ID2 complex [40].

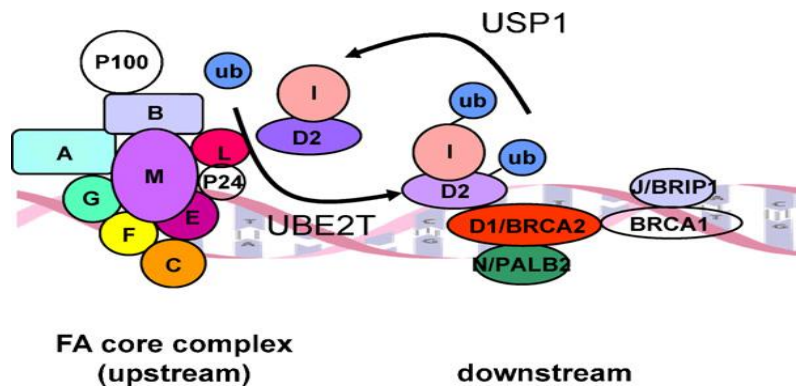

Fig 6: ID2 complex Activation [40]

From the Algorithm we can write the process like:

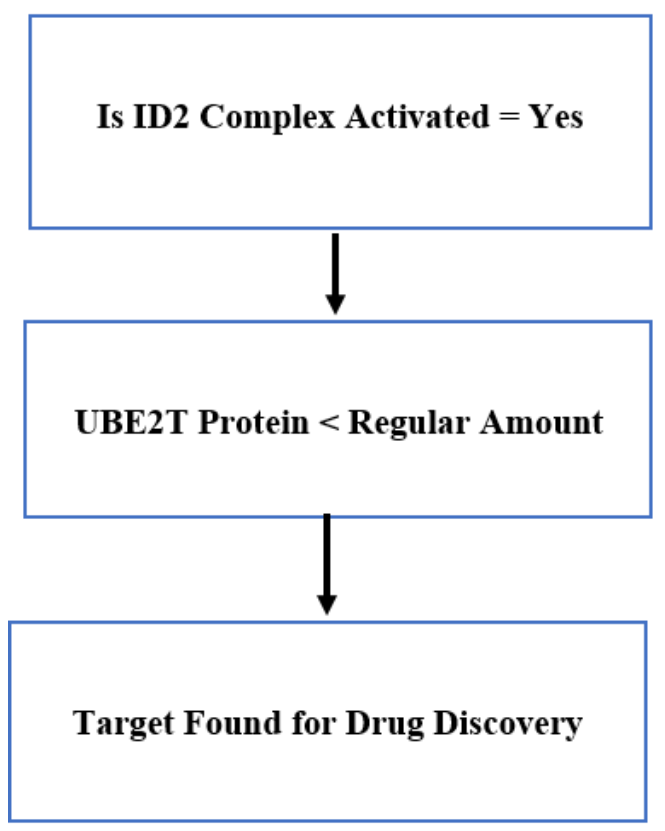

Fig 7: Process of Identifying ID2 Complex Activation

\subsection{XPF-ERCC1 Defection}

A number of nucleases have been suggested to manipulate in ICL repair, including MUS81-EME1, XPF-ERCC1, FAN1, SLX4-SLX1, SNM1A, XPG, and FEN1. Their clear roles have not been understood, but their activity is likely important at several phases of ICL repair, including unhooking, HR, and processing and removal of the unhooked seize. The interaction domain of SLX4 with XPF-ERCC1 is important to confer resistance to ICL inducing agents, and SLX4 colocalizes with XPF-ERCC1 in nuclear foci [45], suggesting that the interaction with XPF is important for the function of SLX4 in ICL repair. Sometimes, XPF-ERCC1 defects causes Fanconi Anemia. 


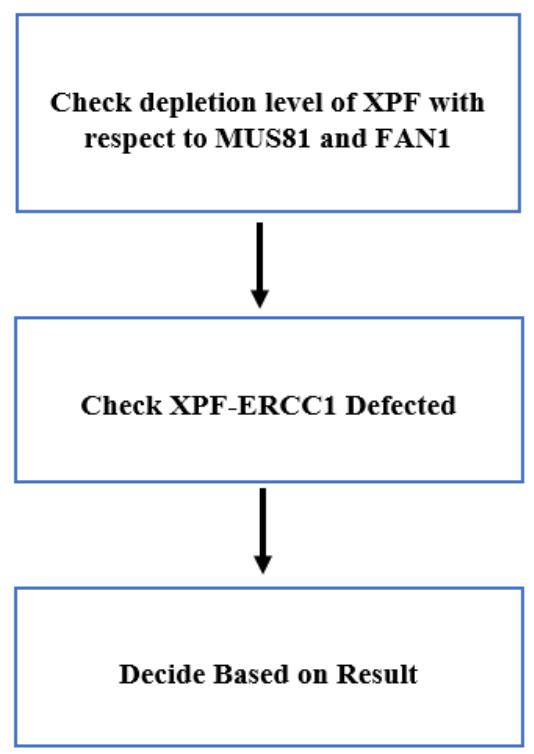

Fig 8: Process of Identifying XPF-ERCC1 Defect

\section{CONCLUSION}

Several malady causes as a result of lack of DNA replication. Fanconi Anemia is the result of a genetic defect in a cluster of proteins responsible for DNA repair which means DNA replication averted. During the DNA replication blockade FA pathway invoke proteins to repair the damaged DNA. This invocation process is very complex. There are three phase which implicates the complex process: (1) Upstream: the FA core complex (2) Nexus: the ID2 complex (3) Downstream: proteins that possess a DNA repair. Disruption in certain protein in this complex process causes Fanconi Anemia like mutation in FA gene, shortage of UBE2T protein, XPFERCC1 defection and abnormal depletion of XPF. So, proposed algorithm which has been described in this study could enhance the solution for target identification of Fanconi Anemia effected people. At long last, it remains space for the future research and development on several fields which can be carried on like identify DNA mutation of Fanconi Anemia using methods of protein structure comparison, ICL recognition of Fanconi Anemia using machine learning tools.

\section{ACKNOWLEDGMENTS}

Our gladness to Almighty and my supervisor for his relentless conduct, encouragement, and endurance, and for giving me the opportunity to do this work.

Eventually, my profound gratefulness and predilection to my parents for their buttress, encouragement, and incessant love.

\section{REFERENCES}

[1] T.M. Schroeder, F. Anschütz,A. Knopp, Spontane chromosomenaberrationen bei familiärer panmyelopathie, Humangenetik 1 (1964) 194-196.

[2] M. Sasaki, Is Fanconi's anaemia defective in a process essential for the repair of DNA cross links? Nature 257 (1975) 501-503.

[3] A.D. Auerbach, S.R. Wolman, Susceptibility of Fanconi's anaemia fibroblasts to chromosome damage by carcinogens, Nature 261 (1976) 494-496.

[4] Gluckman E, Wagner JE. Hematopoietic stem cell transplantation in childhood inherited bone marrow failure syndrome. Bone Marrow Transplant. 2008; 41(2):127-132.

[5] Alter BP. Diagnosis, genetics, and management of inherited bone marrow failure syndromes. Hematology Am Soc Hematol Educ Program. 2007;29-39.

[6] Auerbach AD, Wolman SR. Carcinogen-induced chromosome breakage in Fanconi's anaemia heterozygous cells. Nature. 1978;271:69-71.

[7] Macmillan ML, Wagner JE. Haematopoeitic cell transplantation for Fanconi anaemia - when and how? $\mathrm{Br}$ J Haematol. 2010 Feb 5.

[8] Gluckman E, Devergie A, Schaison G, et al. Bone marrow transplantation in Fanconi anaemia. $\mathrm{Br} \mathrm{J}$ Haematol. 1980;45:557-564.

[9] Sociẻe G, Gluckman E, Raynal B, et al. Bone marrow transplantation for Fanconi anemia using low-dose cyclophosphamide/ thoracoabdominal irradiation as conditioning regimen: chimerism study by the polymerase chain reaction. Blood. 1993;82: 2249-2256.

[10] Polina Stepensky, Michael Y. Shapira, Dmitry Balashov, Pavel Trakhtman, Elena Skorobogatova, Lyudmila Rheingold, Rebecca Brooks, Shoshana Revel-Vilk, Michael Weintraub, Jerry Stein, Alexey Maschan, Reuven Or, Igor B. Resnick Bone Marrow Transplantation for Fanconi Anemia Using FludarabineBased Conditioning, Biol Blood Marrow Transplant 17: 1282-1288 (2011)

[11] C.A. Strathdee, H. Gavish, W.R. Shannon, M. Buchwald, Cloning of cDNAs for Fanconi's anaemia by functional complementation,Nature 356 (1992) 763-767.

[12] J.R. Lo Ten Foe, M.A. Rooimans, L. Bosnoyan-Collins, N. Alon, M. Wijker, L. Parker, J. Lightfoot, M. Carreau, D.F. Callen, A. Savoia, N.C. Cheng, C.G. van Berkel, M.H. Strunk, J.J. Gille, G. Pals, F.A. Kruyt, J.C. Pronk, F. Arwert, M. Buchwald, H. Joenje, Expression cloning of a cDNA for the major Fanconi anaemia gene, FAA, Nat. Genet. 14 (1996) 320-323.

[13] J.P. de Winter, Q. Waisfisz, M.A. Rooimans, C.G.M. van Berkel, L. Bosnoyan- Collins, N. Alon, M. Carreau, O. Bender, I. Demuth, D. Schindler, J.C. Pronk, F. Arwert, H. Hoehn, M. Digweed, M. Buchwald, H. Joenje, The Fanconi anaemia group G gene FANCG is identical with XRCC9, Nat. Genet. 20 (1998) 281-283.

[14] J.P. de Winter, M.A. Rooimans, L. van der Weel, C.G.M. van Berkel, N. Alon, L. Bosnoyan-Collins, J. De Groot, Y. Zhi, Q. Waisfisz, J.C. Pronk, F. Arwert, C.G. Mathew, R.J. Scheper,M.E. Hoatlin, M. Buchwald, H. Joenje, The Fanconi anaemia gene FANCF encodes a novel protein with homology to ROM, Nat. Genet. 24 (2000) 15-16.

[15] Y.J. Machida, Y. Machida, Y. Chen, A.M. Gurtan, G.M. Kupfer, A.D. D'Andrea, A. Dutta, UBE2T is the E2 in the Fanconi anemia pathway and undergoes negative autoregulation, Mol. Cell 23 (2006) 589-596.

[16] S.M. Nijman, T.T. Huang, A.M. Dirac, T.R Brummelkamp, R.M. Kerkhoven, A.D. D'Andrea, R. Bernards, The deubiquitinatingenzymeUSP1regulates the Fanconi anemia pathway, Mol. Cell 17 (2005) 331-339. 
[17] Syed Asif Hassan, Ahmed Hamza Osman. "An Improved Machine Learning Approach to Enhance the Predictive Accuracy for Screening Potential Active USP1/UAF1 Inhibitors", Vol. 8, No. 4, 2017.

[18] Daisy Klein Douwel, Rick A.C.M. Boonen, David T. Long, Anna A. Szypowska, Markus Ra" schle, Johannes C. Walter, and Puck Knipscheer, "XPF-ERCC1 Acts in Unhooking DNA Interstrand Crosslinks in Cooperation with FANCD2 and FANCP/SLX4", available at http://dx.doi.org/10.1016/j.molcel.2014.03.015.

[19] Yi-Ping Phoebe Chen \& Feng Chen, "Identifying targets for drug discovery using bioinformatics", Ther. Targets (2008) 12(4):383-389.

[20] A. D. D'Andrea, M. Grompe, Nat. Rev. Cancer 3, 23 (2003)

[21] W. Wang, Nat. Rev. Genet. 8, 735 (2007).

[22] Johan P. de Winter, Hans Joenje. "The genetic and molecular basis of Fanconi anemia". Department of Clinical Genetics.

[23] The Fanconi Anemia Pathway of DNA Repair and Human Cancer By Vaidehi Krishnan, Lavina Sierra Tay and Yoshiaki Ito DOI: $10.5772 / 59995$

[24] Paweł Mackiewicz Where does bacterial replication start? Rules for predicting the oriC region, Nucleic Acids Research, 2004, Vol. 32, No. 13 3781-3791 ,doi:10.1093/nar/gkh699

[25] A. F. Alpi, P. E. Pace, M. M. Babu, K. J. Patel, Mol. Cell 32, 767 (2008)

[26] I. Garcia-Higuera et al., Mol. Cell 7, 249 (2001).

[27] A. Smogorzewska et al., Cell 129, 289 (2007).

[28] T. Taniguchi et al., Blood 100, 2414 (2002).

[29] Orlando D Schärer, "ERCC1- XPF endonuclease positioned to cut”, DOI 10.15252/embj.201797489.

[30] M. Ben-Yehoyada et al., Mol. Cell 35, 704 (2009).

[31] X. Shen et al., Mol. Cell 35, 716 (2009)

[32] A. Sobeck et al., Mol. Cell. Biol. 26, 425 (2006).

[33] Y. M. Akkari et al., Mol. Genet. Metab. 74, 403 (2001).
[34] M. Raschle et al., Cell 134, 969 (2008).

[35] L. H. Thompson, J. M. Hinz, Mutat. Res. 668, 54 (2009).

[36] Ciccia A, et al. Identification of FAAP24, a Fanconi anemia core complex protein that interacts with FANCM. Molecular cell. 2007;25(3):331-43. [PubMed]

[37] Yan Z, et al. A histone-fold complex and FANCM form a conserved DNA-remodeling complex to maintain genome stability. Molecular cell. 2010;37(6):865-78. [PMC free article] [PubMed]

[38] Singh TR, et al. MHF1-MHF2, a histone-fold-containing protein complex, participates in the Fanconi anemia pathway via FANCM. Molecular cell. 2010;37(6):87986. [PMC free article] [PubMed]

[39] Kim JM, et al. Cell cycle-dependent chromatin loading of the Fanconi anemia core complex by FANCM/FAAP24. Blood. 2008;111(10):5215-22.

[40] K.A. Rickman, F.P. Lach, A. Abhyankar, F.X. Donovan, E.M. Sanborn, J.A. Kennedy, C. Sougnez, S.B. Gabriel, O. Elemento, S.C. Chandrasekharappa, et al. Deficiency of UBE2T, the E2 ubiquitin ligase necessary for FANCD2 and FANCI ubiquitination, causes FA-T subtype of Fanconi Anemia

[41] JPred4: A Protein Secondary Structure Prediction Server". Retrieved 16 July 2015.

[42] Jump up to: a b Drozdetskiy, Alexey; Cole, Chris; Procter, James; Barton, Geoffrey (Apr 16, 2015). "JPred4: a protein secondary structure prediction server". Nucleic Acids Research. 43: W389-W394. PMC 4489285 Freely accessible. PMID 25883141 doi:10.1093/nar/gkv332.

[43] Irina Kufareva and Ruben Abagyan, "Methods of protein structure comparison", doi: 10.1007/978-1-61779-5886_10.

[44] Feng Gao and Chun-Ting Zhang , Ori-Finder: A webbased system for finding oriC $\mathrm{s}$ in unannotated bacterial genomes, BMC Bioinformatics 2008, doi: 10.1186/14712105-9-79.

[45] Gerry P. Crossan et al. Disruption of mouse Slx4, a regulator of structure-specific nucleases, phenocopies Fanconi Anemia, doi: 10.1038/ng.752. 